\title{
Improved molecular toolkit for cAMP studies in live cells
}

Kwan Pyo Hong ${ }^{1}$, Nicholas C Spitzer ${ }^{1}$ and Xavier Nicol ${ }^{1,2^{*}}$

\begin{abstract}
Background: CAMP is a ubiquitous second messenger involved in a wide spectrum of cellular processes including gene transcription, cell proliferation, and axonal pathfinding. Precise spatiotemporal manipulation and monitoring in live cells are crucial for investigation of CAMP-dependent pathways, but existing tools have several limitations.

Findings: We have improved the suitability of CAMP manipulating and monitoring tools for live cell imaging. We attached a red fluorescent tag to photoactivated adenylyl cyclase (PAC $\alpha$ ) that enables reliable visualization of this optogenetic tool for CAMP manipulation in target cells independently of its photoactivation. We show that replacement of CFP/YFP FRET pair with GFP/mCherry in the Epac2-camps FRET probe reduces photobleaching and stabilizes the noise level during imaging experiments.

Conclusions: The modifications of PAC $\alpha$ and Epac2-camps enhance these tools for in vitro CAMP studies in cultured living cells and in vivo studies in live animals in a wide range of experiments, and particularly for long term time-lapse imaging.
\end{abstract}

\section{Background}

cAMP is a major cellular second messenger that activates and integrates multiple intracellular signaling pathways and modulates a large range of cellular processes, including gene transcription [1], cell adhesion and migration [2], and axonal growth and pathfinding [3]. cAMP studies rely on methods to manipulate and monitor cAMP concentrations in live cells. Existing tools have been very useful in identifying cAMP-dependent cellular processes, but have some limitations when it comes to understanding cAMP dynamics and localization in living cells. Forskolin and 3isobutyl-1-methylxanthine (IBMX) are powerful pharmacological compounds enabling the generation of sustained elevations of cAMP. Forskolin directly stimulates most transmembrane adenylyl cyclases [4] and IBMX inhibits cAMP hydrolysis by phosphodiesterases. Recently, the use of photoactivated adenylyl cyclase alpha (PAC $\alpha$ ) from the flagellate Euglena gracilis, synthesizing cAMP in response to blue light, has allowed precise spatiotemporal manipulation of cAMP [5]. It has been attached to GFP for visualization in live cells [6]. However, the excitation wavelength

\footnotetext{
* Correspondence: xavier.nicol@inserm.fr

${ }^{1}$ Neurobiology Section, Division of Biological Sciences, Kavli Institute for Brain and Mind, University of California, San Diego, La Jolla, CA 92093, USA

Full list of author information is available at the end of the article
}

of this visible reporter overlaps with the excitation spectrum of PAC $\alpha$, making it difficult to use this fusion construct for independent $\mathrm{PAC} \alpha$ excitation and reporter imaging.

Monitoring cAMP in live cells has been made possible by the use of FRET probes [7-10]. Epac2-camps is a cAMP indicator that is widely used to monitor cAMP [10] and has been recently improved with a mutation increasing its affinity for cAMP [11]. However, fast photobleaching of the commonly used CFP/YFP FRET pair limits its use in live cell imaging experiments over extended periods of time because the signal-to-noise ratio decreases progressively. The GFP/mCherry FRET pair has been successfully used for cAMP sensors [12], but its photostability and signal-to-noise ratio have not been assessed.

\section{Results}

Independent excitation of PAC $\alpha$ and mCherry in living cells and live animals

The red fluorescent protein mCherry was expected to be an appropriate tag for PACa since its excitation wavelength in the green range of visible light [13] is distinct from PACa excitation by blue and UV light [14] (Figure 1A). A mCherry-PAC $\alpha$ fusion protein was 


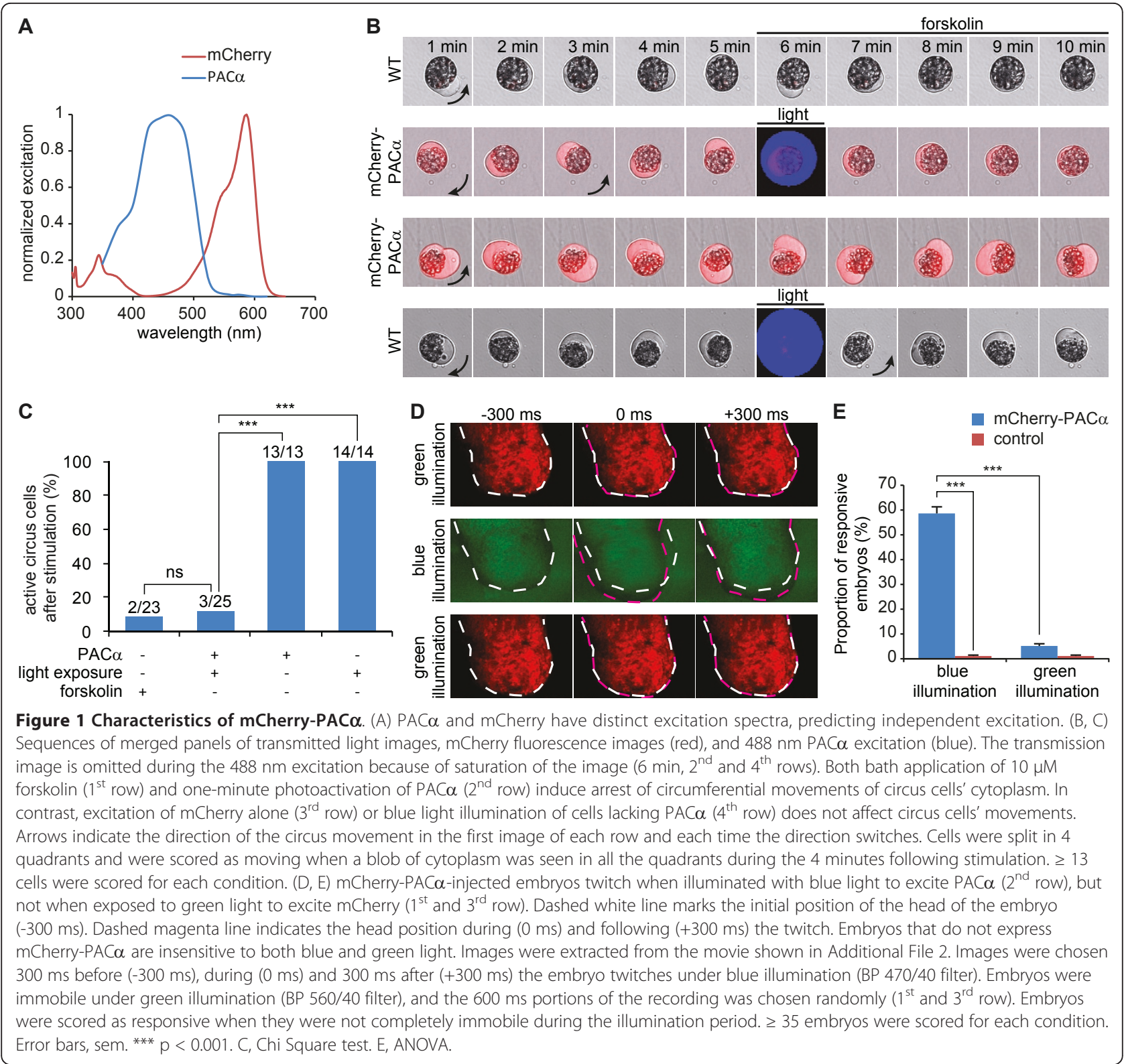

generated using a mutant of PAC $\alpha$ (R330A) that has a limited adenylyl cyclase activity in the dark (G. Nagel, personal communication). mCherry was attached to the N-terminus of PAC $\alpha$ (linker: SGLRSRAQASNSAVDGTA). The fluorescence of mCherry and lightdependent cAMP synthesis of PAC $\alpha$ appear unaffected in the fusion product. mRNA coding for mCherryPAC $\alpha$ was transcribed using the mMessage Ultra kit (Ambion). 1 to $3 \mathrm{ng}$ of mRNA were injected in both blastomeres of 2-cell stage Xenopus laevis embryos, which were incubated in the dark for $24 \mathrm{hr}$ at $23^{\circ} \mathrm{C}$. Dissociated cells from stage 21 embryo neural tubes were plated onto plastic dishes and kept in the dark for $2 \mathrm{hr}$. Cultures from injected but not control animals were fluorescent when illuminated at $561 \mathrm{~nm}$ on a Leica SP5 confocal microscope, revealing the expression of mCherry (Figure 1B). We then developed a bioassay to assess the function of this construct. Circus cells exhibit circular movement of the plasma membrane in cultures from Xenopus neural tubes [15]. Application of $10 \mu \mathrm{M}$ forskolin blocked the circus movements of these cells (Figure $1 \mathrm{~B}$ and $1 \mathrm{C}$ ), demonstrating that a high concentration of cAMP prevents these plasma membrane movements. We next tested the light-dependent cyclase activity of mCherry-PAC $\alpha$ by illuminating mCherry-positive cells at $488 \mathrm{~nm}$. A 1-minute exposure abolished membrane movements. In contrast, mCherry excitation did not affect circus movements and 
mCherry-negative cells did not exhibit plasma membrane movement arrest following stimulation by either wavelength (Figure $1 \mathrm{~B}$ and $1 \mathrm{C}$ and see Additional file 1). We conclude that mCherry-PAC $\alpha$ combines the features of mCherry for cell identification and PAC $\alpha$ for light-sensitive cyclase activity, and that mCherry and PAC $\alpha$ excitation wavelengths are exclusive from each other.

To verify that this construct can be used in vivo, Xenopus embryos injected with mCherry-PAC $\alpha$ mRNA were illuminated with green and blue light in alternation using a fluorescence dissecting microscope with GFP and Texas-red filter cubes. Blue light illumination (excitation filter: BP 470/40) induced embryos to twitch, whereas they remain completely immobile under green light illumination (excitation filter: BP 560/40) (Figure 1D and 1E and see Additional file 2). Uninjected embryos did not exhibit light-induced twitching. Excitation of mCherry did not affect embryos' behavior, confirming the spectral compatibility of mCherry and PAC $\alpha$.

\section{Reduced photobleaching of Epac2-camps using the GFP/ mCherry FRET pair}

In addition to the improvement of PAC $\alpha$ to manipulate cAMP, we modified the FRET pair of the existing Epac2-camps cAMP sensor to improve the stability of its signal-to-noise ratio over longer periods of time. We replaced the CFP/YFP pair by GFP/mCherry, with the same linkers as in the original probe [10]. mCherry and GFP have a low photobleaching rate [16] and constitute an efficient FRET pair [12] with smaller overlap between the emission spectra of the acceptor and the donor (Figure $2 \mathrm{~A}$ ). In addition, we included a mutation in the cAMP binding domain (K405E) that has been shown to reduce the $\mathrm{Kd}$ of the probe from $900 \mathrm{nM}$ to $300 \mathrm{nM}$ [11]. To demonstrate the improvement of the sensor, mRNA coding for membrane-targeted Epac2-camps including either CFP/YFP (pm-Epac2-camps-CFP/YFP) or GFP/mCherry (pm-Epac2-camps-GFP/mCherry) was transcribed and expressed in Xenopus laevis neural tube cultures as described for mCherry-PAC $\alpha$. Cultures were continuously superfused and three images per minute were acquired with $442 \mathrm{~nm}$ laser excitation and filters adapted for each FRET pair (CFP: $460 \mathrm{~nm}-500 \mathrm{~nm}$; YFP: $525 \mathrm{~nm}-580 \mathrm{~nm}$; GFP: $480 \mathrm{~nm}-550 \mathrm{~nm}$; mCherry: 590 $\mathrm{nm}-700 \mathrm{~nm}$ ). $10 \mu \mathrm{M}$ forskolin induced a similar FRET ratio increase for pm-Epac2-camps-CFP/YFP and pmEpac2-camps-GFP/mCherry, revealing an appropriate FRET response to cAMP elevation for both probes (Figure $2 \mathrm{~B}$ ). To assess the resistance to photobleaching, cells were continuously excited at $442 \mathrm{~nm}$. CFP and YFP intensity decreased rapidly whereas GFP and mCherry showed reduced photobleaching (Figure $2 \mathrm{C}$ and $2 \mathrm{D}$ ). Although $442 \mathrm{~nm}$ is not at the peak of excitation of
GFP, a forskolin-induced increase of the GFP:mCherry ratio can be detected using this excitation wavelength (Figure 2B). Exciting GFP with a $442 \mathrm{~nm}$ laser line has the advantage of minimizing the direct excitation of the acceptor (mCherry) that perturbs FRET measurements when longer excitation wavelengths are used. To assess the noise level, we monitored the standard deviation of CFP:YFP and GFP:mCherry FRET ratio changes over 40 frames during the 1000 frames imaging period. The standard deviation of pm-Epac2-camps-GFP/mCherry was stable throughout the measurement period, while the standard deviation of pm-Epac2-camps-CFP/YFP increased over time (Figure $2 \mathrm{E}$ and $2 \mathrm{~F}$ ). The progressive increase of the noise level over time may be due to the increased relative contribution of the noise to the fluorescence measurement of each channel: the fluorescence intensity decreases because of photobleaching, but the noise level is not affected. Consequently, the noise level of the donor:acceptor ratio is higher after photobleaching.

\section{Conclusion}

In summary, we have generated an improved toolkit for cAMP studies. mCherry-PAC $\alpha$ allows spatiotemporal control of cAMP in living cells after identification of cells expressing it. Localized illumination of a cell is likely to increase cAMP concentration locally. Further development of PAC $\alpha$ may include its targeting to subcellular compartments to go beyond the limit of precision of optical stimulation and achieve cAMP manipulation bearing closer resemblance to physiological signals. It would be useful to ensure for each experimental condition that the cyclase activity of PAC $\alpha$ in the dark does not affect intracellular signaling $[5,17]$. To limit cAMP synthesis without light exposure, we used a mutated PAC $\alpha$ (R330A) that has a limited cyclase activity in the dark (G. Nagel, personal communication). This was enough to avoid perturbation of circus cells movement by the cyclase activity of PAC $\alpha$ in the dark. In case an extremely low dark activity is needed, bPAC, a bacterial light-sensitive adenylyl cyclase, could be used at the cost of less stringent temporal control of cAMP signaling [17].

$\mathrm{pm}$-Epac2-camps-GFP/mCherry has greater photostability than pm-Epac2-camps-CFP/YFP and a lower noise level after extended periods of imaging. However GFP and mCherry make a less effective FRET pair than CFP and YFP, and its use may be beneficial only for FRET experiments requiring an extended period of imaging. Versions of CFP and YFP (mTurquoise and Venus respectively) with increased photostability are now available and make an efficient FRET pair for cAMP sensors [18]. Testing its noise level stability would allow comparison of the behaviour of GFP/mCherry and mTurquoise/ Venus as FRET pairs for prolonged experiments. The 


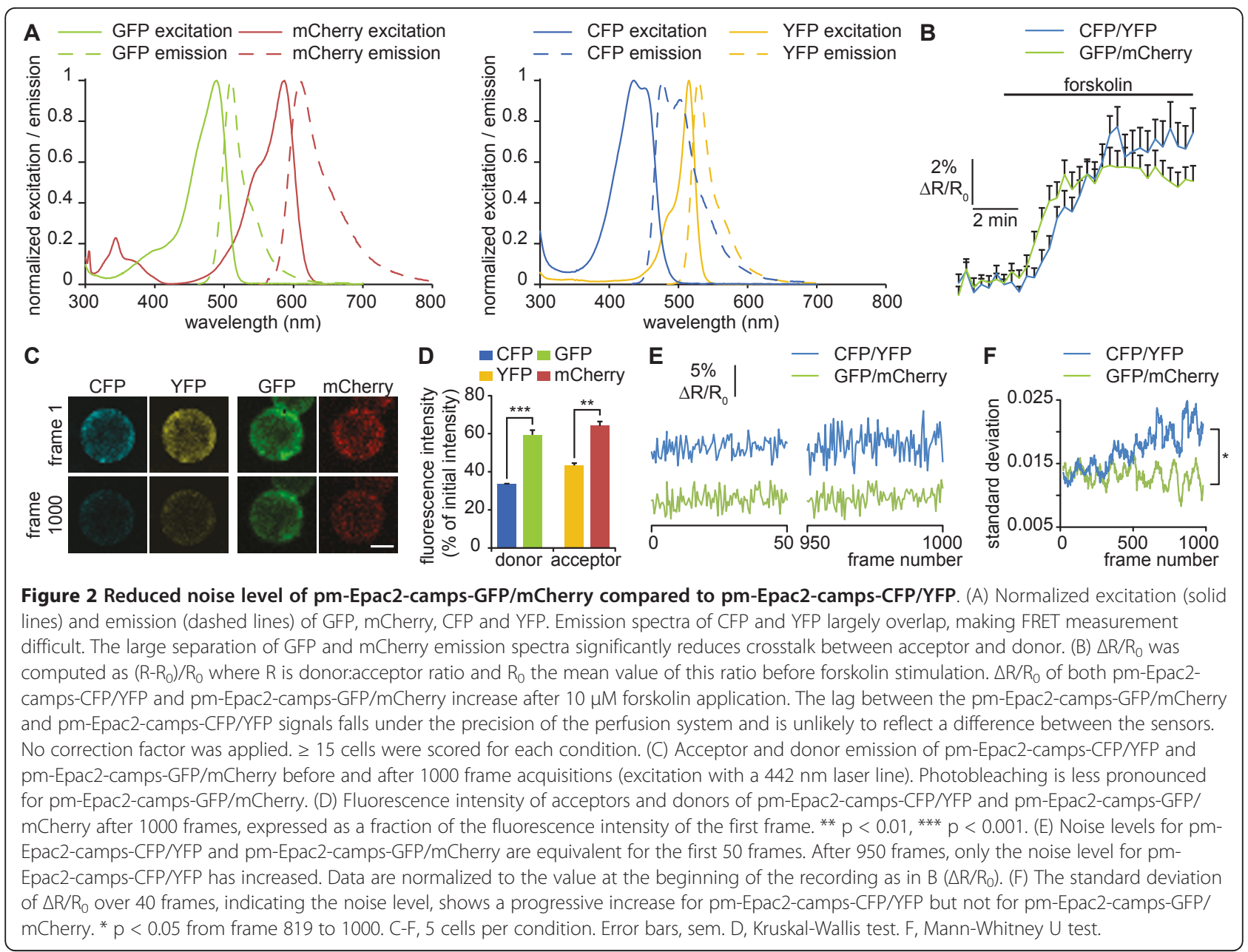

sensor described here has the advantage over the mTurquoise/Venus probe [18] of sensitivity to lower concentrations of cAMP, because it is an Epac2-based sensor including a mutation that reduces its $\mathrm{Kd}$ [11]. It would be useful to compare it to the Epac1-based sensor using mTurquoise/Venus, with the higher Kd for cAMP $[9,10,18]$.

The use of both tools in the same cell is not yet possible due to the overlap of excitation wavelengths, but further improvements may include the shift of PACa excitation towards the UV to avoid wavelength conflict with the FRET probe excitation, in combination with the switch of the mCherry tag to a longer wavelength fluorescent protein such as mKate to avoid the overlap of emission between the PAC $\alpha$ tag and the FRET acceptor.

\section{Additional material}

Additional file 1: Photoactivation of PAC $\alpha$ mimics forskolin-induced arrest of circus cells in culture. In circus cells in cultures from the $X$. laevis neural tube, blebs of plasma membrane propagate around the cell circumference with a period of several minutes (Olson, 1996). Forskolin stimulation of endogenous adenylyl cyclases in control cells arrests this blebbing movement (top left panel). Blue light (488 $\mathrm{nm}$ ) irradiation of mCherry-PAC $\alpha$-expressing cells mimics the effect of forskolin stimulation (top right panel). In contrast, blebbing movements are not affected in non-blue light-irradiated mCherry-PAC $\alpha$-expressing cells (bottom left panel) or in control cells illuminated at $488 \mathrm{~nm}$ (bottom right panel).

Additional file 2: Photoactivation of PAC $\alpha$ induces $X$. laevis embryo twitching. mCherry-PAC $\alpha$-injected embryos twitch when illuminated with blue light to excite PAC $\alpha$, but not when exposed to green light to excite mCherry.

\section{Acknowledgements}

We thank Dr M. Roe for the gift of pm-Epac2-camps, and Dr G. Nagel for the gift of PAC $\alpha$. We are grateful to members of our lab for thoughtful discussion. This work was supported by a Fondation pour la Recherche Médicale fellowship and a Marie Curie International Outgoing Fellowship within the 7th European Community Framework Programme to X.N., and by NIH NS15918 to N.C.S.

\section{Author details}

${ }^{1}$ Neurobiology Section, Division of Biological Sciences, Kavli Institute for Brain and Mind, University of California, San Diego, La Jolla, CA 92093, USA. 2INSERM, U839, Université Paris 6, Institut du Fer à Moulin, 17 rue du Fer à Moulin, 75005 Paris, France. 


\section{Authors' contributions}

$\mathrm{KPH}$ and XN carried out the experiments. NCS and XN conceived the experimental design. $\mathrm{KPH}, \mathrm{NCS}$ and XN wrote the manuscript. All authors have read and approved the final manuscript.

\section{Competing interests}

The authors declare that they have no competing interests.

Received: 6 April 2011 Accepted: 20 July 2011 Published: 20 July 2011

\section{References}

1. Sands WA, Palmer TM: Regulating gene transcription in response to cyclic AMP elevation. Cell Signal 2008, 20:460-466

2. Howe AK: Regulation of actin-based cell migration by CAMP/PKA. Biochim Biophys Acta 2004, 1692:159-174.

3. Piper M, van Horck F, Holt C: The role of cyclic nucleotides in axon guidance. Adv Exp Med Biol 2007, 621:134-143.

4. Laurenza A, Sutkowski EM, Seamon KB: Forskolin: a specific stimulator of adenylyl cyclase or a diterpene with multiple sites of action? Trends Pharmacol Sci 1989, 10:442-447.

5. Schröder-Lang S, Schwärzel M, Seifert R, Strünker T, Kateriya S, Looser J, Watanabe M, Kaupp UB, Hegemann P, Nagel G: Fast manipulation of cellular cAMP level by light in vivo. Nat Methods 2007, 4:39-42.

6. Weissenberger S, Schultheis C, Liewald JF, Erbguth K, Nagel G, Gottschalk A: PACa-an optogenetic tool for in vivo manipulation of cellular CAMP levels, neurotransmitter release, and behavior in Caenorhabditis elegans. J Neurochem 2011, 116:616-625.

7. Zhang J, Ma Y, Taylor SS, Tsien RY: Genetically encoded reporters of protein kinase A activity reveal impact of substrate tethering. Proc Natl Acad Sci USA 2001, 98:14997-15002.

8. Zaccolo M, De Giorgi F, Cho CY, Feng L, Knapp T, Negulescu PA, Taylor SS, Tsien RY, Pozzan T: A genetically encoded, fluorescent indicator for cyclic AMP in living cells. Nat Cell Biol 2000, 2:25-29.

9. Ponsioen B, Zhao J, Riedl J, Zwartkruis F, van der Krogt G, Zaccolo M, Moolenaar WH, Bos JL, Jalink K: Detecting cAMP-induced Epac activation by fluorescence resonance energy transfer: Epac as a novel cAMP indicator. EMBO Rep 2004, 5:1176-1180.

10. Nikolaev VO, Bünemann M, Hein L, Hannawacker A, Lohse MJ: Novel single chain CAMP sensors for receptor-induced signal propagation. J Biol Chem 2004, 279:37215-37218

11. Norris RP, Ratzan WJ, Freudzon M, Mehlmann LM, Krall J, Movsesian MA Wang $H$, Ke H, Nikolaev VO, Jaffe LA: Cyclic GMP from the surrounding somatic cells regulates cyclic AMP and meiosis in the mouse oocyte. Development 2009, 136:1869-1878.

12. van der Krogt GNM, Ogink J, Ponsioen B, Jalink K: A comparison of donoracceptor pairs for genetically encoded FRET sensors: application to the Epac cAMP sensor as an example. PLoS ONE 2008, 3:e1916.

13. Shaner NC, Campbell RE, Steinbach PA, Giepmans BNG, Palmer AE, Tsien RY: Improved monomeric red, orange and yellow fluorescent proteins derived from Discosoma sp. red fluorescent protein. Nat Biotechnol 2004, 22:1567-1572.

14. Yoshikawa S, Suzuki T, Watanabe M, Iseki M: Kinetic analysis of the activation of photoactivated adenylyl cyclase (PAC), a blue-light receptor for photomovements of Euglena. Photochem Photobiol Sci 2005, 4:727-731.

15. Olson EC: Onset of electrical excitability during a period of circus plasma membrane movements in differentiating Xenopus neurons. J Neurosci 1996, 16:5117-5129.

16. Tramier M, Zahid M, Mevel J-C, Masse M-J, Coppey-Moisan M: Sensitivity of CFP/YFP and GFP/mCherry pairs to donor photobleaching on FRET determination by fluorescence lifetime imaging microscopy in living cells. Microsc Res Tech 2006, 69:933-939.

17. Stierl M, Stumpf $P$, Udwari D, Gueta R, Hagedorn R, Losi A, Gärtner W, Petereit L, Efetova M, Schwarzel M, Oertner TG, Nagel G, Hegemann P: Light modulation of cellular cAMP by a small bacterial photoactivated adenylyl cyclase, bPAC, of the soil bacterium Beggiatoa. J Biol Chem 2011, 286:1181-1188.

18. Klarenbeek JB, Goedhart J, Hink MA, Gadella TWJ, Jalink K: A mTurquoiseBased CAMP Sensor for Both FLIM and Ratiometric Read-Out Has Improved Dynamic Range. PLOS ONE 2011, 6:e19170. doi:10.1186/1756-0500-4-241

Cite this article as: Hong et al:: Improved molecular toolkit for CAMP studies in live cells. BMC Research Notes 2011 4:241.

\section{Submit your next manuscript to BioMed Central and take full advantage of:}

- Convenient online submission

- Thorough peer review

- No space constraints or color figure charges

- Immediate publication on acceptance

- Inclusion in PubMed, CAS, Scopus and Google Scholar

- Research which is freely available for redistribution

Submit your manuscript at www.biomedcentral.com/submit
C Biomed Central 